\title{
Entecavir and Low Genetic Barrier Antiviral Agents for Hepatocellular Carcinoma in Hepatitis B Viral Cirrhosis: Propensity Score Matching
}

\author{
Tao Li\#, Yundong Qu\#, Yan Wang, Chunlei Lin, Baohua Yang and Lei Wang
}

\begin{abstract}
Objective: To compare the reduction of hepatocellular carcinoma (HCC) risk between long-term treatment of entecavir and low genetic barrier antiviral agents in hepatitis B virus (HBV)-related cirrhotic patients.

Study Design: An observational study.

Place and Duration of Study: Department of Infectious Diseases and Hepatology, the Second Hospital of Shandong University, Jinan, China, from October 2008 to October 2016.

Methodology: HBV-related cirrhotic patients with antiviral treatment for at least 12 months were consecutively included. Propensity score matching analysis was performed to improve comparability of the data from both entecavir group and the control group. Log-rank test was used to compare influence of various nucleos(t)ide analogs (NAs) for incidence of HCC. Independent risk factors were estimated by multivariable Cox proportional hazards models.

Results: The total cohort included $207 \mathrm{HBV}$-related cirrhotic patients, of which 83 patients were treated with entecavir initially. The present study found no statistical difference for the incidence of HCC between entecavir group and the control group in the total cohort $(p=0.525)$. However, the difference became statistically significant $(p=0.014)$ after propensity score matching. Number needed to treat (NNT) were 8 patients, 6 patients and 3 patients at years 2,3 and 4 , respectively. Multivariable Cox regression in propensity score matching cohort revealed older age (HR: 1.066, $p=0.041)$, NAs of low generic barrier (HR: 6.944, $p=0.016)$, NAs resistance (HR: 3.648, $p=0.041)$, and lower platelet counts (<80x109/L) (HR: 6.718, $\mathrm{p}=0.009$ ) as independent risk factors for HCC incidence.

Conclusion: Entecavir is more efficient in reducing the incident HCC risk for HBV-related cirrhotic patients in comparison to low genetic barrier NAs.
\end{abstract}

Key Words: Hepatocellular carcinoma, Liver cirrhosis, Hepatitis B virus, Entecavir, Comparative effectiveness research.

\section{INTRODUCTION}

About 240 million people worldwide have been chronically infected with hepatitis $B$ virus (HBV). The infection rate is disturbingly high in the Asian Pacific region. ${ }^{1}$ Chronic hepatitis $\mathrm{B}(\mathrm{CHB})$ infection increases the risk of cirrhosis, hepatic decompensation, and hepatocellular carcinoma (HCC). Previous researches reveal that around $15-40 \%$ of the infected people suffer from severe diseases caused by hepatitis B. 1 To prevent tragic consequences of hepatitis $B$ infection such as cirrhosis, hepatic decompensation, end-stage liver disease, HCC and death, antiviral therapy is widely used. 1

Within the past $10-20$ years, five oral nucleos(t)ide analogs (NAs) have been proved as efficient antiviral drugs. According to previous studies, lamivudine is able to significantly reduce mortality and to prevent the

Department of Infectious Diseases and Hepatology, the Second Hospital of Shandong University, Jinan 250033, China

Correspondence: Dr. Lei Wang, Department of Infectious Diseases and Hepatology, the Second Hospital of Shandong

University, 247 Beiyuan Road, Jinan 250033, China

E-mail:wlsdeygbk@163.com

Received: July 31, 2018; Accepted: October 30, 2018

\# These authors contributed equally. occurrence of $\mathrm{HCC}$ in patients with $\mathrm{CHB}$ compared with no antiviral treatment. ${ }^{2}$ Entecavir, a relatively new antiviral agent with high genetic barrier, has been proved to suppress HBV DNA replications effectively with relatively low drug-resistance rate. ${ }^{3}$ It is also reported that the incidence of HCC in CHB patients has been significantly reduced by entecavir. ${ }^{4}$ Although low genetic barrier agents (lamivudine, adefovir and telbivudine) have not been recommended as preferred regimens by current guidelines, they are still widely used considering the cost and availability of NAs, especially in some parts of Asia and Africa. ${ }^{5}$ Even different genetic barriers, and several studies did not show any obvious difference for the incidence of $\mathrm{HCC}$ in patients with $\mathrm{CHB}$ or cirrhosis when they were treated with lamivudine or entecavir. 6-8 It has also been noticed that some studies showed the HCC suppression effect of entecavir was greater than that of lamivudine. 4 The inconsistent conclusions make clinicians puzzled whether higher genetic barriers are equal to more significant HCC suppression effect. Meanwhile, the question which merits further discussion is that whether current evidence is conclusive enough to persuade cirrhotic patients with acceptable antiviral effect caused by low generic barrier NAs to be treated with high genetic barrier NAs. Further studies deriving from clinical practice are greatly needed. The usage of 
propensity score matching makes it possible to reduce bias due to confounding factors in real-world observational studies. Furthermore, uptill now, most of similar studies have focused on the comparison for incidence of HCC with entecavir and lamivudine, without other low genetic barrier drugs such as adefovir and telbivudine, which is not in conformity with the clinical practice.

The purpose of the present study was to compare the reduction of $\mathrm{HCC}$ risk between long-term treatment of entecavir and low genetic barrier drugs (lamivudine, adefovir and telbivudine) in HBV-related cirrhotic patients by a propensity score matching analysis.

\section{METHODOLOGY}

This study was a retrospective collection of observational data. Consecutive HBV-related cirrhotic patients with antiviral therapy for at least 12 months were retrospectively evaluated and enrolled to Department of Infectious Diseases and Hepatology, the Second Hospital of Shandong University, Jinan, China between October 2008 and October 2016. All included patients were treatment naïve and were initially treated with NAs (lamivudine, adefovir, telbivudine or entecavir). Patients initially treated with lamivudine plus adefovir or lamivudine plus interferon were also included. It was also required that the patients being selected show hepatitis $B$ surface antigen ( $\mathrm{HBsAg}$ ) positive for at least 6 months before the commencement of the antiviral therapy. To ensure the accuracy of the results, those who were co-infected with other virus (e.g. hepatitis C virus, human immunodeficiency virus) or combined with other liver diseases (e.g. autoimmune hepatitis, druginduced-liver disease), and patients with HCC during the first year of NAs therapy were excluded. The 1-year interval was established to minimize the risk of both inclusion of preexisting HCC for analyses and inappropriate attribution of antiviral effect.8,9 This study protocol followed guidelines of the Helsinki Declaration and has been approved by the Institutional Review Board of the Second Hospital of Shandong University. Patients were regularly monitored every 3 to 6 months and the following inspection items were performed: liver biochemistry examination, international normalized ratio (INR), $\alpha$-fetoprotein (AFP) level, serum HBV DNA, hepatitis $B$ e antigen $(\mathrm{HBeAg})$ and imaging examinations (ultrasound, CT, or MRI). The observational outcome of this study was the occurrence of HCC in entecavir group and control group (patients treated with low genetic barrier drugs: lamivudine, adefovir and telbivudine. Lamivudine plus adefovir or lamivudine plus interferon were also included). HCC was defined as a tumor with a maximum diameter more than $2 \mathrm{~cm}$ and typical features of HCC with arterial hypervascularity and venous or delayed phase washout using CT or MRI, or nodules of 1-2 cm and typical features of HCC with two coincident imaging techniques (CT and MRI).
Serum alanine aminotransferase (ALT), aspartate aminotransferase (AST), bilirubin were measured by Beckman CX7 Chemistry Analyzer (Beckman Coulter, CA, USA) and original reagents. $\mathrm{HBsAg}$, antibody against $\mathrm{HBsAg}$ (anti-HBs), hepatitis $\mathrm{B}$ e antigen ( $\mathrm{HBeAg}$ ) and antibody against $\mathrm{HBeAg}$ (anti-HBe) were detected with a commercial radioimmunoassay kit (Abbott Laboratories, Abbott Park, IL, USA). Serum HBV DNA levels were detected by using real-time PCR with suitable reagents (Sinomd Gene, Beijing, China). The lowest detection limit of HBV DNA was 500 copies/ml.

The propensity score matching analysis was applied to improve the comparability of the data from both entecavir group and control group. A propensity score of each patient was calculated by using logistic regression, covariates included in the multivariable logistic regression analysis, model were age, gender, status of HBeAg, HBV DNA, ALT, AST, bilirubin, AFP, platelet count, INR, history of alcohol intake, family history of HCC, NAs resistance and follow-up duration. We used 1:1 nearest-neighbor matching and the match tolerance between two participants was 0.05 . Patients failed to match were excluded. Log-rank test was performed to compare the influence of different NAs on the incidence of HCC.

The normality of continuous variables was evaluated by Kolmogorov-Smirnov test. Continuous variables were expressed as mean \pm standard deviation (SD) or median interquartile range (IQR) and were compared with t-test or Mann-Whitney U-test as appropriate. Categorical data was compared with $\chi^{2}$ test or Fisher's exact test. Logarithmic transformation was performed at HBV DNA level for further statistical analysis. Survival curves were generated by Kaplan-Meier method. Independent risk factors were evaluated by multivariable Cox proportional hazard models (Forward LR method). Number needed to treat NNT to prevent 1 additional occurrence of HCC comparing entecavir with low genetic barrier drugs was also calculated according to the method of Altman et al.10 A p-value <0.05 (two-tailed) implied statistically significance. All statistical analyses were performed by using IBM SPSS Statistics Version 22.0 (IBM Corp., Armonk, NY, USA).

\section{RESULTS}

The total cohort included 207 HBV-related cirrhotic patients, of which 83 patients were treated with entecavir initially (entecavir group). For the other 124 cirrhotic patients (control group: patients treated with NAs of lower genetic barrier), the initial NAs were lamivudine in 42 patients, adefovir in 74 patients, telbivudine in five patients, lamivudine plus adefovir in two patients and lamivudine plus interferon in one patient. Patients in the entecavir group were typical of lower incidence of NAs resistance $(p<0.001)$ and shorter follow-up duration $(p<0.001)$ in comparison to those in the control group (Table I). 
HCC occurred in $30(14.5 \%)$ of the 207 cirrhotic patients during a median of 31 (IQR 19-49) months' follow-up. Among those patients, 7 patients $(8.4 \%)$ were from entecavir group and 23 patients $(18.5 \%)$ were from the control group (Table II). For patients in entecavir group and patients in control group (treated with low genetic barrier drugs), the cumulative incidence rate of HCC at months 24, 36, and 48 by Kaplan-Meier analysis were $8.7 \%, 12.6 \%, 12.6 \%$ and $5.5 \%, 10.0 \%, 16.2 \%$, respectively. Log-rank test revealed no statistical difference for the incidence rate of HCC between the above two groups ( $p=0.525$, Figure 1$)$.

As for baseline characteristics, an imbalance existed between entecavir group and the control group. To correct the above imbalance, a propensity score matching analysis was applied according to several baseline characteristics. The propensity score matching cohort yielded 112 (56 pairs) of cirrhotic patients. The median follow-up duration in entecavir group and that in the control group were 28.0 (IQR 18.0-36.0) months and 25.0 (IQR 18.0-35.0) months, respectively $(p=0.731)$. The incidence of NAs resistance were $7.1 \%$ (4 patients) and $19.6 \%$ (11 patients) in the two groups $(p=0.052)$. We believed that the baseline characteristics were comparable between entecavir group and the control group after the performance of propensity score matching (Table I).

In the propensity score matching cohort, 13 (11.6\%) cirrhotic patients developed HCC. Among them, 2 patients $(3.6 \%)$ belonged to entecavir group and 11 patients (19.6\%) belonged to the control group (Table II). For patients in entecavir group and patients in control group, the cumulative incidence rate of $\mathrm{HCC}$ at months 24,36 , and 48 were $2.3 \%, 6.7 \%, 6.7 \%$ and $14.5 \%$,

Table I: Characteristics of the total cohort and propensity score-matched cohort.

\begin{tabular}{|c|c|c|c|c|c|c|}
\hline & \multicolumn{3}{|c|}{ Total cohort } & \multicolumn{3}{|c|}{ Propensity score-matched cohort } \\
\hline & $\begin{array}{c}\text { Entecavir } \\
(n=83)\end{array}$ & $\begin{array}{c}\text { Drugs with } \\
\text { lower generic } \\
\text { barrier }(n=124)\end{array}$ & $p$-value & $\begin{array}{l}\text { Entecavir } \\
(n=56)\end{array}$ & $\begin{array}{c}\text { Drugs with } \\
\text { lower generic } \\
\text { barrier }(n=56)\end{array}$ & p-value \\
\hline Age (years) & $48.8 \pm 11.1$ & $48.3 \pm 12.1$ & 0.779 & $48.2 \pm 11.2$ & $48.2 \pm 13.0$ & 0.988 \\
\hline \multicolumn{7}{|l|}{ Gender } \\
\hline Male $(n, \%)$ & $59(71.1 \%)$ & $84(67.7 \%)$ & 0.610 & $39(69.6 \%)$ & $41(73.2 \%)$ & 0.676 \\
\hline Female $(n, \%)$ & $24(28.9 \%)$ & $40(32.3 \%)$ & & $17(30.4 \%)$ & $15(26.8 \%)$ & \\
\hline \multicolumn{7}{|l|}{$\mathrm{HBeAg}$} \\
\hline Positive & $53(63.9 \%)$ & $70(56.5 \%)$ & 0.288 & $33(58.9 \%)$ & $34(60.7 \%)$ & 0.847 \\
\hline Negative & $30(36.1 \%)$ & $54(43.5 \%)$ & & $2(41.1 \%)$ & $22(39.3 \%)$ & \\
\hline HBV DNA $\left(\log _{10}\right.$ copy/ml) & $5.8 \pm 1.5$ & $5.7 \pm 1.2$ & 0.375 & $5.6 \pm 1.5$ & $5.9 \pm 1.3$ & 0.216 \\
\hline $\operatorname{ALT}(\mathrm{U} / \mathrm{L})$, median $(\mathrm{IQR})$ & $59.0(34.0-119.0)$ & $60.0(39.0-113.0)$ & 0.760 & $57.0(31.0-97.0)$ & $73.0(45.0-141.0)$ & .033 \\
\hline $\mathrm{AST}(\mathrm{U} / \mathrm{L})$, median $(\mathrm{IQR})$ & $60.0(45.0-110.0)$ & $62.0(46.0-125.0)$ & 0.564 & $57.0(42.0-106.0)$ & $62.0(48.0-135.0)$ & 0.164 \\
\hline Bilirubin (umol/L), median (IQR) & $30.2(19.2-48.4)$ & $33.1(18.3-58.1)$ & 0.730 & $28.3(19.0-40.8)$ & $33.8(20.2-56.6)$ & 0.253 \\
\hline Platelet count (109/L), median (IQR) & $91.0(57.0-119.0)$ & $74.0(52.3-112.3)$ & 0.215 & $75.0(55.0-103.0)$ & $82.0(47.0-112.0)$ & 0.928 \\
\hline INR, median (IQR) & $1.16(1.05-1.36)$ & $1.20(1.03-1.47)$ & 0.579 & $1.18(1.04-1.34)$ & $1.20(1.04-1.40)$ & 0.880 \\
\hline $\operatorname{AFP}(\mathrm{ng} / \mathrm{ml})$, median (IQR) & $9.4(4.9-50.4)$ & $11.4(4.2-43.8)$ & 0.878 & $8.0(4.2-40.4)$ & $12.1(5.1-48.1)$ & 0.231 \\
\hline \multicolumn{7}{|l|}{ History of: } \\
\hline Alcohol intake (n, \%) & $22(26.5 \%)$ & $32(25.8 \%)$ & 0.911 & $15(26.8 \%)$ & $16(28.6 \%)$ & 0.833 \\
\hline No alcohol intake (n, \%) & $61(73.5 \%)$ & $92(74.2 \%)$ & & & $41(73.2 \%)$ & $40(71.4 \%)$ \\
\hline \multicolumn{7}{|l|}{ Family history of } \\
\hline $\operatorname{HCC}(\mathrm{n}, \%)$ & $8(9.6 \%)$ & $7(5.6 \%)$ & 0.277 & $5(8.9 \%)$ & $4(7.1 \%)$ & .000 \\
\hline No HCC (n, \%) & $75(90.4 \%)$ & $117(94.4 \%)$ & & $51(91.1 \%)$ & $52(92.9 \%)$ & \\
\hline \multicolumn{7}{|l|}{ NAs resistance } \\
\hline Yes (n, \%) & $5(6.0 \%)$ & $32(25.8 \%)$ & $<0.001$ & $4(7.1 \%)$ & $11(19.6 \%)$ & 0.052 \\
\hline No $(n, \%)$ & $78(94.0 \%)$ & $92(74.2 \%)$ & & $52(92.9 \%)$ & $45(80.4 \%)$ & \\
\hline $\begin{array}{l}\text { Follow-up duration, } \\
\text { months, median (IQR) }\end{array}$ & $22.0(17.0-34.0)$ & $40.0(26.0-60.0)$ & $<0.001$ & $28.0(18.0-36.0)$ & $25.0(18.0-35.0)$ & 0.731 \\
\hline
\end{tabular}

Table II: Treatment strategies in control group (patients treated with NAs of lower genetic barrier).

\begin{tabular}{l|cc|cc}
\hline \multirow{2}{*}{ Treatment strategies } & \multicolumn{2}{|c|}{ Total cohort $(\mathrm{n}, \%)$} & \multicolumn{2}{|c}{ Propensity score-matched cohort $(\mathrm{n}, \%)$} \\
\cline { 2 - 5 } & $\begin{array}{c}\text { No. of patients } \\
(\mathrm{n}=124)\end{array}$ & $\begin{array}{c}\text { No. of patients with HCC } \\
(\mathrm{n}=23)\end{array}$ & $\begin{array}{c}\text { No. of patients } \\
(\mathrm{n}=56)\end{array}$ & $\begin{array}{c}\text { No. of patients with HCC } \\
(\mathrm{n}=11)\end{array}$ \\
\hline Lamivudine & $42(33.9 \%)$ & $9(39.1 \%)$ & $21(37.5 \%)$ & $5(45.5 \%)$ \\
Adefovir & $74(59.7 \%)$ & $13(56.5 \%)$ & $28(50.0 \%)$ & $5(45.5 \%)$ \\
Telbivudine & $5(4.0 \%)$ & $1(4.3 \%)$ & $4(7.1 \%)$ & $1(9.1 \%)$ \\
Lamivudine plus adefovir & $2(1.6 \%)$ & $0(0.0 \%)$ & $2(3.6 \%)$ & $0(0.0 \%)$ \\
Lamivudine plus interferon & $1(0.8 \%)$ & $0(0.0 \%)$ & $1(1.8 \%)$ & $0(0.0 \%)$ \\
\hline
\end{tabular}

NAs = Nucleos(t)ide analogs; HCC = Hepatocellular carcinoma. 


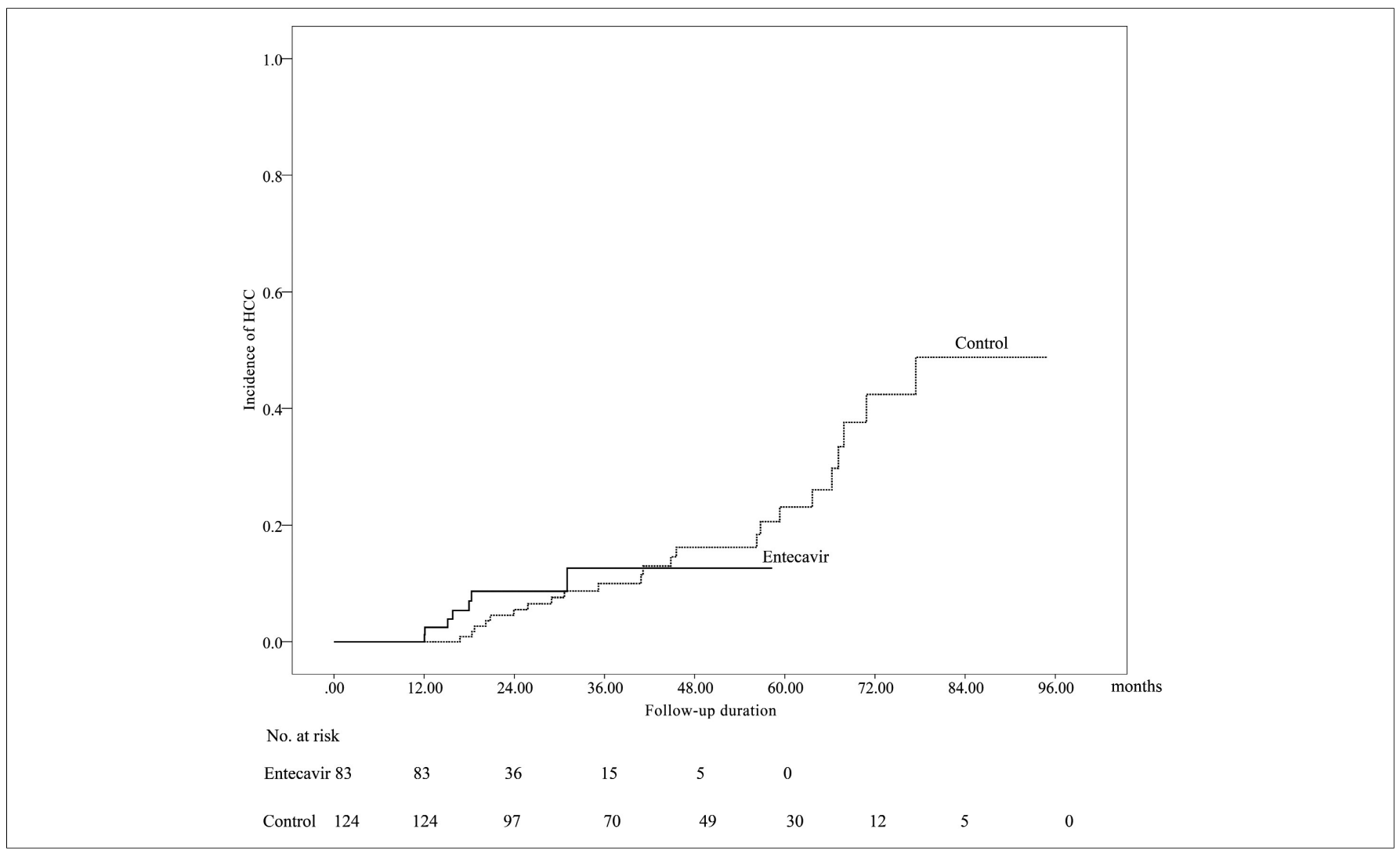

Figure 1: Cumulative incidence of HCC for entecavir group and control group in the total cohort (207 patients).

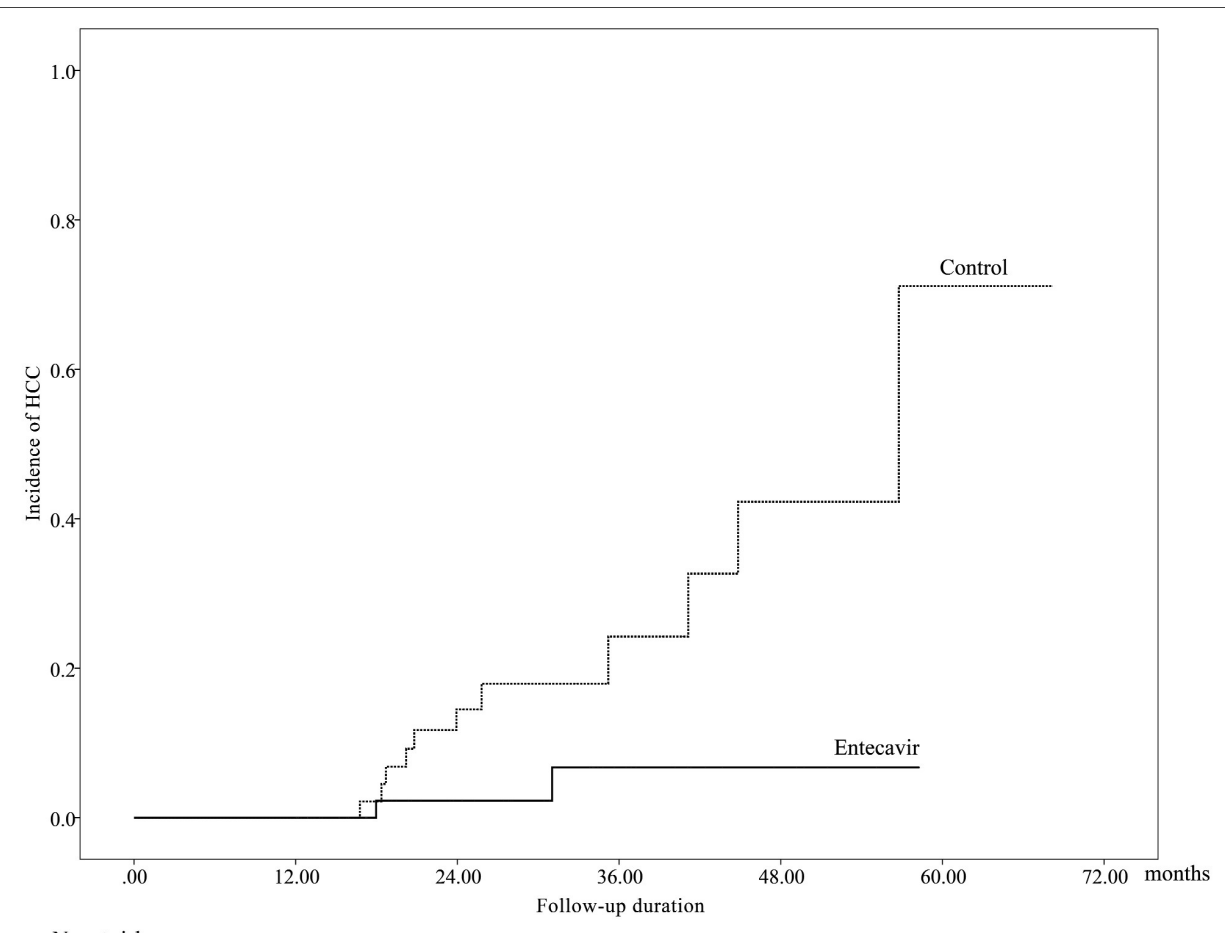

No. at risk

$\begin{array}{lllllll}\text { Entecavir } 56 & 56 & 33 & 14 & 5 & 0 & \\ \text { Control } 56 & 56 & 31 & 12 & 5 & 1 & 0\end{array}$

Figure 2: Cumulative incidence of HCC for entecavir group and control group in the propensity score matching cohort (112 patients). 
$24.2 \%, 42.3 \%$, respectively, yielding NNT of 8 patients, 6 patients and 3 patients at years 2, 3 and 4, respectively. The difference between the two groups for the incidence rate of $\mathrm{HCC}$ was statistically significant according to the results of Log-rank test ( $p=0.014$, Figure 2$)$. The risk of $\mathrm{HCC}$ was also evaluated by multivariable Cox regression in this propensity score matching cohort adjusted for different NAs. Older age (HR: $1.066,95 \% \mathrm{Cl} 1.002-$ $1.134, p=0.041)$, NAs of low generic barrier (HR: 6.944, $95 \% \mathrm{Cl} 1.427-34.483, \mathrm{p}=0.016$ ), NAs resistance (HR: $3.648,95 \% \mathrm{Cl} 1.057-12.595, \mathrm{p}=0.041)$ and lower platelet counts (<80x109/L) (HR: 6.718, 95\% Cl 1.598-28.244, $\mathrm{p}=0.009$ ) were identified as independent risk factors for $\mathrm{HCC}$ incidence.

\section{DISCUSSION}

The present study compared the different HCC suppressive effect between low genetic barrier NAs (lamivudine, adefovir, telbivudine) and a relatively higher genetic barrier agents (entecavir) for HBV-related cirrhotic patients. There was no significant difference for the incidence rate of HCC between entecavir group and control group in total cohort $(p=0.525$, Figure 1$)$. To redress the imbalance of baseline characteristics (especially for follow-up duration and NAs resistance) between the two groups, a propensity score matching method was performed to eliminate the baseline difference. As a result, a sample pool consisting of 112 (56 pairs) patients was established to offset the margin of error and to deliver reliable results. The final results indicated that HCC incidence in patients treated with entecavir was significantly lower than that in patients treated with low genetic barrier drugs, which was a strong indication of the advantage of high genetic barrier agents (entecavir). Low NNT (3, 6 and 8 at years 2, 3 and 4 , respectively) also revealed similar conclusions. The present study identified the following risk factors related to the incidence of $\mathrm{HCC}$ during NAs treatment: older age, lower platelet counts, drug resistance, and low genetic barrier agents.

Previous researchers have revealed that there is a causal link between HBV infection and development of HCC. Studies concluded a 5-year cumulative HCC incidence rate of $3.3 \%$ for chronic HBV patients, and 21.2 - 59\% among cirrhotic patients before antiviral therapy were widely used. ${ }^{11,12}$ Although antiviral therapy was found to be effective in eliminating the risk of progression and postponing the outbreak of HCC for CHB patients, incidences of HCC were still high according to results of several cohorts. ${ }^{9,13-15}$ Risk factors of HCC identified by other studies include older age, male gender, cirrhotic status, AST, HBsAg, HBeAg, genotype $\mathrm{C}$, diabetes mellitus, family history of $\mathrm{HCC}$ and alcohol consumption.8,9,16-20 The present study also drew the similar conclusions as mentioned above. But only four risk factors were got after propensity score matching. The reason might be that all of these patients were at the stage of cirrhosis, which was different from some published studies.

Do more convincing antiviral effect and higher genetic barrier deliver the same effect in terms of reducing the risk of HCC? Answers to this question have never been consistent. Kobashi et al. showed no significant difference in the incidence of HCC between entecavir group and lamivudine group. ${ }^{7}$ However, the difference for follow-up duration between the two groups was a main limitation. Lim et al. reported that there was no significant difference in the incidence of HCC between entecavir group and lamivudine group with the method of propensity score matching in CHB patients. ${ }^{8}$ Baseline characteristics of low genetic barrier NAs (lamivudine, adefovir, telbivudine) and high genetic barrier agents (entecavir) were comparable upon the application of propensity score matching method in the present study. Based on this, the study came to the conclusion that entecavir was more effective than low genetic barrier drugs in clinical treatment (Figure 2). This outcome was conformed to the findings of Hosaka et al. ${ }^{4}$ Better yet, patients with NAs resistance in the present cohort received rescue therapy, which were not performed by Hosaka et al. because of historical limitations.4,6 To enhance the accuracy of the results, the present study included treatment strategies with low genetic barrier NAs besides lamivudine. Some strategies (e.g. telbivudine, lamivudine plus adefovir) were able to deliver more effective HBV DNA suppression and/or less occurrence of resistance than lamivudine ${ }^{1}$, which strengthened credibility of the conclusions.

Antiviral therapy is related to a virologic response and consequent reduction of hepatic necroinflammation. ${ }^{21,22}$ A greater decrease of serum HBV DNA levels $(<104$ copies $/ \mathrm{mL}$ ) during patients' follow-up process indicate a lower incident HCC risk.23,24 The authors believe that the virological breakthrough of HBV and consequent elevated alanine aminotransferase can be at least partially attributed to a higher risk of HCC during followup process. ${ }^{6}$ Kurokawa et al. reported that an average of 5 years' treatment with lamivudine reduced the incidence of HCC in HBV-related cirrhotic patients. ${ }^{25}$ However, $51 \%$ of included patients in this study developed YMDD mutation during follow-up process. In the present study, incidences of NAs resistance were $7.1 \%$ (4 patients) in entecavir cohort and 19.6\% (11 patients) in low genetic barrier NAs cohort $(p=0.052)$. It was found the occurrence risk of HCC in patients with NAs resistance was 3.65 times than that in patients without drug resistance, revealing that the reduction of HCC incidence for patients with cirrhosis required NAs with higher genetic barrier. In addition, it was noticed that occurrence risk of $\mathrm{HCC}$ in low genetic barrier NAs cohort was 6.94 times than that in patients with 
entecavir, calling for the necessity of NAs with more potent antiviral effect. Therefore, even if cirrhotic patients have achieved acceptable antiviral effect after treatment of low genetic barrier NAs, their antiviral strategies are recommended to be switched to high genetic barrier NAs.

The development of a propensity score matching system to evaluate the treatment effects for cirrhotic patients may be helpful in determining the most suitable initial treatment strategy in clinical practice. Although a relatively small number of included patients after propensity score matching, the present study represented outcomes in real-world characteristics rather than in restrictive clinical trial. The current study is featured by the specificity to cirrhosis stage, the real property of included patients and the comparable baseline characteristics, which enhance the accuracy of the results. The research highlights the effect of high genetic barrier drug (entecavir) for the decrease of HCC incidence with comparison to low genetic barrier NAs, providing evidence for the current guidelines. However, data for comparison of tenofovir and low genetic barrier NAs is still lacking and further inquires are needed in future.

\section{CONCLUSION}

In this retrospective observational study, entecavir significantly reduces the incidence of $\mathrm{HCC}$ comparing to low genetic barrier NAs (lamivudine, adefovir, telbivudine) for patients with HBV-related cirrhosis. Therefore, NAs with more potent antiviral activity and higher genetic barrier should be recommended strongly by clinicians, if possible, even for cirrhotic patients who have achieved acceptable antiviral effect caused by low generic barrier NAs.

Acknowledgements: This study was funded by the National Science and Technology Major Project (No. 2013ZX10004902), the Project of Science and Technology Development Plan of Shandong Province (No. 2013GSF11808), and Youth Fund of the Second Hospital of Shandong University (Grant number: Y2014010012).

\section{REFERENCES}

1. Sarin SK, Kumar M, Lau GK, Abbas Z, Chan HL, Chen CJ, et al. Asian-Pacific clinical practice guidelines on the management of hepatitis B: A 2015 update. Hepatol Int 2016; 10:1-98.

2. Liaw YF, Sung JJ, Chow WC, Farrell G, Lee CZ, Yuen H, et al. Lamivudine for patients with chronic hepatitis $\mathrm{B}$ and advanced liver disease. N Engl J Med 2004; 351:1521-31.

3. Chang TT, Lai CL, Kew Yoon S, Lee SS, Coelho HS, Carrilho FJ, et al. Entecavir treatment for up to 5 years in patients with hepatitis B e antigen-positive chronic hepatitis B. Hepatology 2010; 51:422-30.

4. Hosaka T, Suzuki F, Kobayashi M, Seko Y, Kawamura Y, Sezaki $\mathrm{H}$, et al. Long-term entecavir treatment reduces hepatocellular carcinoma incidence in patients with hepatitis $B$ virus infection. Hepatology 2013; 58:98-107.
5. WHO. Guidelines for the prevention, care and treatment of persons with chronic hepatitis B infection (2015). http:// www.who.int/hiv/pub/hepatitis/hepatitis-b-guidelines-policy/en/ (2018-07-15)

6. Hiramatsu N, Yamada R, Takehara T. The suppressive effect of nucleos(t)ide analogue treatment on the incidence of hepatocellular carcinoma in chronic hepatitis B patients. J Gastroenterol Hepatol 2016; 31:546-52.

7. Kobashi H, Miyake Y, Ikeda F, Yasunaka T, Nishino K, Moriya A, et al. Long-term outcome and hepatocellular carcinoma development in chronic hepatitis B or cirrhosis patients after nucleoside analog treatment with entecavir or lamivudine. Hepatol Res 2011; 41:405-16.

8. Lim YS, Han S, Heo NY, Shim JH, Lee HC, Suh DJ. Mortality, liver transplantation, and hepatocellular carcinoma among patients with chronic hepatitis B treated with entecavir vs lamivudine. Gastroenterology 2014; 147:152-61.

9. Kim SS, Hwang JC, Lim SG, Ahn SJ, Cheong JY, Cho SW. Effect of virological response to entecavir on the development of hepatocellular carcinoma in hepatitis B viral cirrhotic patients: Comparison between compensated and decompensated cirrhosis. Am J Gastroenterol 2014; 109:1223-33.

10. Altman DG, Andersen PK. Calculating the number needed to treat for trials where the outcome is time to an event. BMJ 1999; 319:1492-5.

11. Lo KJ, Tong MJ, Chien MC, Tsai YT, Liaw YF, Yang KC, et al. The natural course of hepatitis $B$ surface antigen-positive chronic active hepatitis in Taiwan. J Infect Dis 1982; 146: 205-10.

12. Kato Y, Nakata K, Omagari K, Furukawa R, Kusumoto Y, Mori I, et al. Risk of hepatocellular carcinoma in patients with cirrhosis in Japan. Cancer 1994; 74:2234-8.

13. Papatheodoridis GV, Lampertico P, Manolakopoulos S, Lok A. Incidence of hepatocellular carcinoma in chronic hepatitis B patients receiving nucleos $(\mathrm{t})$ ide therapy: a systematic review. J Hepatol 2010; 53:348-56.

14. Tawada A, Kanda T, Imazeki F, Yokosuka O. Prevention of hepatitis $B$ virus-associated liver diseases by antiviral therapy. Hepatol Int 2016; 10:574-93.

15. Bi J, Zhang Z, Qin E, Hou J, Liu S, Liu Z, et al. Nucleoside analogs treatment delay the onset of hepatocellular carcinoma in patients with HBV-related cirrhosis. Oncotarget 2017; 8: 96725-31.

16. Zoutendijk R, Reijnders JG, Zoulim F, Brown A, Mutimer DJ, Deterding $\mathrm{K}$, et al. Virological response to entecavir is associated with a better clinical outcome in chronic hepatitis $B$ patients with cirrhosis. Gut 2013; 62:760-5.

17. Gordon SC, Lamerato LE, Rupp LB, Li J, Holmberg SD, Moorman AC, et al. Antiviral therapy for chronic hepatitis B virus infection and development of hepatocellular carcinoma in a US population. Clin Gastroenterol Hepatol 2014; 12:885-93.

18. Arends P, Sonneveld MJ, Zoutendijk R, Carey I, Brown A, Fasano $\mathrm{M}$, et al. Entecavir treatment does not eliminate the risk of hepatocellular carcinoma in chronic hepatitis B: Limited role for risk scores in Caucasians. Gut 2015; 64:1289-95.

19. Rapti I, Hadziyannis S. Risk for hepatocellular carcinoma in the course of chronic hepatitis $B$ virus infection and the protective effect of therapy with nucleos(t)ide analogues. World $\mathrm{J}$ Hepatol 2015; 7:1064-73. 
20. Papatheodoridis GV, Idilman R, Dalekos GN, Buti M, Chi H, van Boemmel $\mathrm{F}$, et al. The risk of hepatocellular carcinoma decreases after the first 5 years of entecavir or tenofovir in Caucasians with chronic hepatitis B. Hepatology 2017; 66: 1444-53.

21. Chang TT, Gish RG, de Man R, Gadano A, Sollano J, Chao YC, et al. A comparison of entecavir and lamivudine for $\mathrm{HBeAg}$ positive chronic hepatitis B. N Engl J Med 2006; 354:1001-10.

22. $\mathrm{Yu} \mathrm{JH}$, Lee JI. Current role of transient elastography in the management of chronic hepatitis B patients. Ultrasonography 2017; 36:86-94.

23. Chen CF, Lee WC, Yang HI, Chang HC, Jen CL, lloeje UH, et al.
Changes in serum levels of HBV DNA and alanine aminotransferase determine risk for hepatocellular carcinoma. Gastroenterology 2011; 141:1240-8.

24. Nam JY, Chang Y, Cho H, Kang SH, Cho YY, Cho EJ, et al. Delayed viral suppression during antiviral therapy is associated with increased hepatocellular carcinoma rates in HBeAg-positive high viral load chronic hepatitis B. J Viral Hepat 2018; 25:552-60.

25. Kurokawa M, Hiramatsu N, Oze T, Yakushijin T, Miyazaki M, Hosui $\mathrm{A}$, et al. Long-term effect of lamivudine treatment on the incidence of hepatocellular carcinoma in patients with hepatitis $B$ virus infection. J Gastroenterol 2012; 47:577-85.

.......... 